\title{
The Mode of Urban Renewal Base on the Smart City Theory under the Background of New Urbanization
}

\begin{abstract}
The Smart City, coordinated development of economy, ecology and society, is the future of cities. Urban renewal is an important way to build the Smart City. Based on the Smart City theory, the authors propose the " 12345 " model of urban renewal, which means developing a tailored proposal of the city and citizen, and building "one city management system, two supporting safeguard measures, three information infrastructure platforms, four urban management modes, and five application service systems." The city will gain the ability of self-adjustment and self-improvement. Smart urban renewal model not only solves the problems of the city at this stage, but also promotes the all-round development of the city, and access to sustainable development.
\end{abstract}

Keywords: urban renewal, smart city, self-adjustment, self-improvement, mode

\section{Introduction}

Along with the advancement of the national new urbanization, problems about urban ecology, resources and society have become increasingly serious. Urban renewal, which is the important means to keep the necessary functions and to solve the current problems of the cities, has attracted great attention in recent years. However urban renewal in China has still been in the groping stage. Owing to old planning thinking and building methods being neither perfect nor normative, urban renewal and reconstruction have many problems: planning is not forward-looking and doesn't match the needs of development of the city, and redundant constructions are widespread; complete demolition and building bring destructive effects to the ecological and the cultural

Manuscript received June 18, 2015; accepted September 10, 2015

Yi-hua Mao (ه), Hong-yu Li, Qin-rui Xu

College of Civil Engineering and Architecture, Zhejiang University, Hangzhou 310058, China

Email: zjumyh@163.com environment; public participation is not enough and conflicts occur in the distribution between public interests and social interests; implementation of panning is not enough, and there are still disadvantages concerning the function and the transportation after the community is updated. The popular pattern of urban renewal is widely criticized, and new ideas about urban development are needed.

There is the suggestion that many present problems in the development of urban construction, largely come from the lack of the ability of constant self-adjustment (Abdoullaev, 2011). Therefore, on the basis of the concept of sustainable development, IBM put forward the concept of "smarter earth" in November 2008, and triggered worldwide great mass fervor about the Smart City construction (Hollands, 2008). Many scholars at home and abroad studied the concept of the Smart City. Deakin, Wright and others put forward ideas or visions of the architecture of the Smart City based on technology (Deakin \& AI Waer, 2011; Xu, Wu \& Chen, 2012; Wright \& Steventon, 2004). Wu and Yang interpreted the connotation of the Smart City from the Angle of the digital city (Wu \& Yang, 2010). But the study did not involve the analysis and consideration on urban society, ecology, management and so on.

From a macro point of view, the Smart City is the integration of the concepts of the ecological city, the digital city, the knowledge-based city and the innovative city which emphasized the all-round development of "economy, ecology and society." What's more, the development of the city and its ability of constant self-adjustment and self-improvement was crucial. Specifically, the Smart City asks for self-growth vigor, namely under the accurate orientation and long-term development planning, the Smart City can realize self-adjustment and self-optimization as to promote sustainable economic development, to improve the ecological environment, to provide digital and intelligent services for residents, to better the quality of material life and spiritual life, and to enhance the sense of happiness. According to the real-time status and problems, the Smart city is the new direction of future urban renewal reconstruction and development. It is an important task to 
clear the new requirements of the Smart City development and construction, to formulate construction guidelines and standardize the construction in the field of urban construction in the future.

\section{The connotation and mode of smart urban renewal and reconstruction}

\subsection{The Smart City is not only a digital city}

In recent years, the Smart City construction has been carried out in many cities at home and abroad, but most of them are simply "armed" existing urban areas with information technology. In Beijing, the Smart City is defined as: integrating information which is closely related with residents' lives by using a new generation information technology to realize that the living elements are digital, networked, intelligent, interactive and cooperative, in order to enhance residents' quality of life (Beijing Social Office, 2013). It is difficult for a city to be called the Smart City only through the integration of information technology in the city. It can only be called digital city or intelligent city. The Smart Cities should apply advanced management concepts and information technology to all aspects of a city, such as construction, management, development, to optimize the allocation of resources systematically and realize the self-perfection and coordinated development of urban functions.

\subsection{The theory model of the Smart City}

In the system of the Smart City, urban residents' demand is the foundation; information technology is the means; the think-tank which is composed of the city policy heads, industry experts and representatives of local residents is the center of control. Information technology means, such as the Internet, cloud computing (providing information) is to promote sound operation and sustainable development of the city (see Figure 1). The Smart City system provides intelligence services to residents, and immediately provide urban statue to the government for their smart decision.

\subsection{The train of thought of smart urban renewal and} reconstruction

Urban renewal can be divided into the city hardware update such as construction of buildings, public infrastructure, and city software updates such as the management mode, supporting services. According to the concept of the Smart City, the connotation of smartness oriented urban renewal should include "people-oriented-development-leading - wisdom guiding." (1) People-oriented, means that city update should respect residents' willingness, meet the demand of service, and improve the urban quality of life. (2) Development-leading, means that urban renewal must promote coordinated development of economy, ecology and environment. (3) Wisdom guiding, which requires embodying the wisdom ideas in the urban renewal design, construction and later operation, is the basis of the peopleoriented. Its objective is to promote comprehensive and balanced city development. Wisdom is its features and advantages.

The development of the urban renewal mode can be summarized as traditional pattern which simply takes measures for the specific problem. Then, it develops into the Smart City mode which manages city by information technology and resolves problems as a whole.

Different from traditional model and intelligent city model, wisdom oriented urban renewal mode should consider wisdom as a new requirement of city, aiming at some problems such as the planning, construction and management, putting forward the solution which reflects the connotation of "people-oriented development wisdom guided."

In a clear positioning on the basis of urban development,

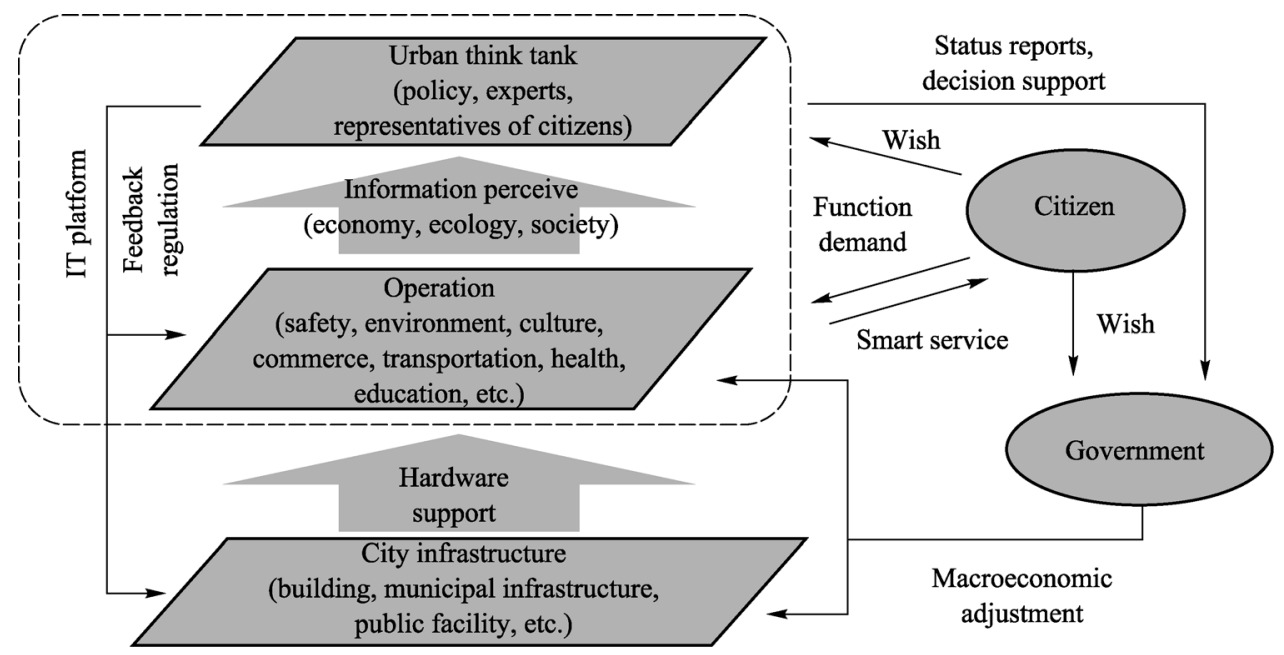

Figure 1. The theoretical model of the Smart City. 
wisdom urban renewal planning should be formulated, considering the comprehensive economic, ecological and social development needs. The objective is to achieve coordinated development of economy and environment, and enhance residents' quality of life.

\section{Urban renewal and reconstruction of the "12345" mode under the background of new urbanization}

Under the background of the new urbanization, urban renewal and reconstruction should solve problems in large and medium-sized cities, realizing the transformation of wisdom. The Smart City construction should be based on the full application of information technology, to improve the existing problems, reflect the connotation of "peopleoriented - development-leading - wisdom guiding," and realize the optimization of resource allocation, urban management and urban decision-making.

Therefore, the smart urban renewal and reconstruction model can be summarized as " 12345 " mode, namely the construction of the Smart City should aim at "one city management system, two supporting safeguard measures, three information infrastructure platforms, four urban management modes, five application service systems" (see Figure 2).

\subsection{One city management system}

The Smart City is different from the traditional city, which relies on the smart management system to adjust the relations among the urban design, construction, management and operation, and realizes self-growth. Therefore, a set of the Smart City management system should be designed first.

Before renewal, the urban need to analyze current problems and form a think-tank, which consists of urban policy sets, industry experts and representatives of local residents. Then, the think-tank, on the basis of investigating and analyzing, needs to demonstrate the Smart City construction and make the strategic planning. Through combining the characteristics of the city, it constructs suitable guidelines and standards for evaluation of the Smart City. The management system includes the construction mechanism and evaluation of the Smart City.

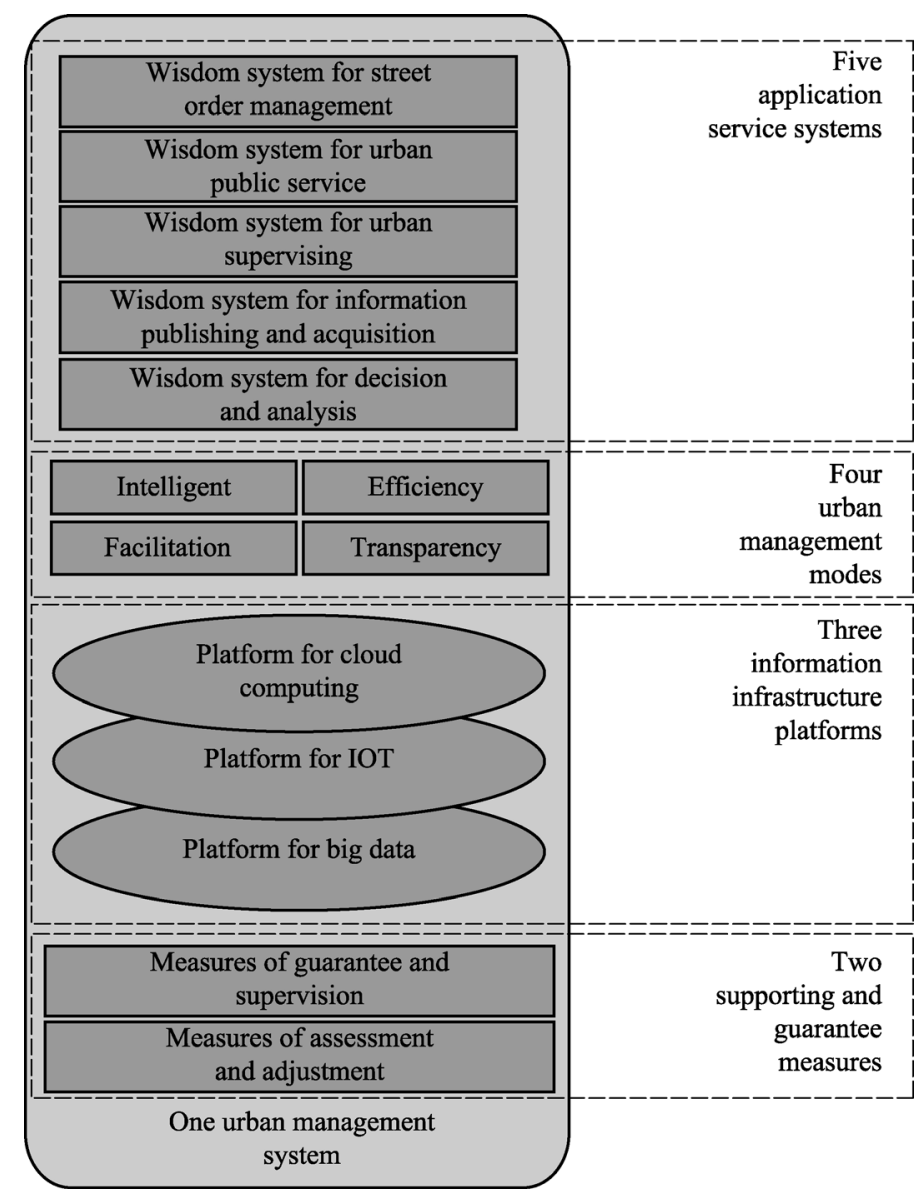

Figure 2. The "12345" mode for city renewal and reconstruction. 


\subsection{Two supporting safeguard measures}

A clear aim, a scientific and reasonable update scheme, an intelligence management system, a smart community management and social service idea, and eco-friendly city hardware facilities, are all needed in the process of urban renewal and reconstruction. Also, it is necessary to have a strong security and supervision to ensure the normal operation. Therefore, as an indispensable part of urban renewal, two supporting safeguard measures, policy supervision and macro regulation, are needed during the reconstruction of the Smart City.

\subsubsection{Security regulation}

Urban renewal should be backed by policy guarantee and regulation, so it must be equipped with strong policy support and supervision system in the whole process of planning, construction, management and development. When we make the update planning, the project should be ensured that it formed after democratic appraisal and expert reasoning and promote the development based on the principle of people-oriented and environment harmonious. Also, policy guidelines are needed to ensure that the project cannot only improve the present situation and the residents' life of the updated regions, but also protect the ecological environment and cultural landscape. At the construction phase, it is necessary to have corresponding supervision measures and that the reconstruction can be in strict accordance with the planning program. Moreover, on the project site, it should supervise the transportation of construction materials, noise, waste emissions, and any other step which may impact on the surrounding environment. In the operation and management of the area, it also needs corresponding policies and regulatory system to protect the normal operation of smart community, such as facilities protection, service system maintenance, corresponding laws and regulations related smart transportation.

\subsubsection{Evaluation and regulation mechanism}

Assessment of regulating mechanism is the compass of the Smart City construction. In the process of urban development, as affected by various factors, the urban economic, social, environmental development goals may be deviation from what expected; unbalance development exists. So, it is necessary to build macro evaluation and regulation mechanism, to monitor and analyze the indicators of urban development on a regular basis, to make adjustment, and to ensure that the construction and development of cities don't drift off the course.

\subsection{Three information infrastructure platforms}

As a foundation for information technology, cloud computing platform, the internet of things platform and data sharing platform, which could provide support for resource allocation, information awareness and publishing, self-regulation of the Smart City, should be set up in the construction of the Smart City.

\subsubsection{Cloud computing platform}

It mainly provide technical support for service delivery of urban construction, management and operation, analytical decision making through constructing a cloud computing infrastructure as a service (IaaS), platform as a service (PaaS) and software as a service (SaaS).

\subsubsection{Internet of things platform}

Constructing the awareness and network platform of the internet system, which combined with urban geographic information system (GIS), radio frequency identification (RFID), GPS, sensing technology, can mainly help us perceive the data of city and provide technical support for urban intelligent identification, intelligent deployment,and data acquisition.

\subsubsection{Data sharing platform}

First, through establishing the data sharing platform, collecting the date delivered by the awareness platform of the internet of things system and receiving the decision made by cloud computing, the platform can collect and release information timely, and provide data support and network resources for the various functions of the city. And then, the platform may realize the function of city data sorting, information release and resource sharing.

\subsection{Four urban management modes}

Management intelligence, work efficiency, service facilitation, and transparent decisions are four demands of the Smart City management model.

Through full intelligent analysis and intelligent adjustment, the intelligent management mode is able to deal with the problems in a timely and optimally manner during the operation.

With resource integration and greatly improved coordination and collaborative capability of the urban operation, efficient management mode can optimize the existing evaluation model and improve the social efficiency.

Facilitative management mode means, optimizing the government service function, building the convenient way of communication between government and citizens, simplifying the process, and providing long-term service and emergency response mechanism, improving the service function of the city.

Transparent management mode, that means, establishing 
an open, fair and impartial decision model, releasing information through the data sharing platform, collecting the aspirations of the citizens, and publishing development plan, and finally realizing the goal of people-oriented decision system of urban development.

\subsection{Five application service systems}

To achieve the four types of urban management mode, we need to develop five matched application service systemintelligent street order management system, urban public service system, urban intelligent management system, information disclosure and acquisition system, intelligent analysis and decision-making system.

Intelligent street order management system can realize intelligence and regulation of urban traffic order. When the system perceives an increased traffic flow, it will feedback to the cloud system as soon as a certain threshold is reached, and through system operation and overall analysis by experts, a set of traffic adjustment plan can be selected. All the plans are designed to regulate the whole areas' traffic, such as increasing the width of road, or changing a road to a one-way street.

When the system perceives a decreased landscaping area caused by air pollution or natural disasters, through the cloud computing and analysis, it can make supplemented or recreated scheme to guarantee the balance of urban ecological environment. When the system perceives the good local economic development momentum and the surplus area of a certain part, through the cloud computing, it can fine-tune the original functional partition, and make it no longer limit the overall development of the city.

Urban public service system could better connect with all aspects of the city and easily provide the residents with intelligent social services. Also, it can intelligently response and coordinate basic social service needs such as business, health, culture and education of the public, and improve resource utilization.

Urban smart management system monitors indicators of economic, ecology and society, timely make adjustment and guidance, to promote the coordinated development of the three. Specifically, the smart management can be reflected in resource allocation, development guidance and urban security. In the daily running of the Smart City, the management system could help resources distributed in demand, lead traffic volume in different time periods and different areas, efficiently response to city security such as urban crime, fire, emergency treatment. With urban development, when some resources and elements within the region are no longer able to meet the development needs of the city, the system will help overall allocation and manage the resources and solve the limitation of development.

Corresponding with the city management mode of transparent decision-making, information disclosure and collection system is a kind of application service. Based on the three major platforms, and network interactive, mobile applications as the carrier, the system is developed to increase public participation in urban management and interactivity.

Intelligent analysis and decision system is responsible for analyzing the indicators which come from urban development and measuring the phase of the urban development, and aims to provide the reference sources of information for an urban think tank to make adjustment program.

\section{Conclusions}

As the new type of urbanization is advocated by the country in the new period, the Smart City is the direction of the future urban development. In developing period, most of large and medium-sized cities solve the city problems and realize the smart construction through urban renewal. Therefore, exploring the Smart Cities' renewal mode is of great significance.

After making clear expectations of the urban development and coordinating the relationship between economic, ecological and social, a set of scientific and reasonable planning of urban renewal can be established under the basis of democratic appraisal and expert reasoning. Based on the Smart City theory, the paper proposes the " 12345 " model of urban renewal, which cannot only provide residents with intelligence services, manage and respond to all kinds of problems in urban construction and operation, but also make tuning adjustments with government decision-making according to the development of the city state and promote urban sustainable development.

\section{References}

Abdoullae, A. (2011). A smart world: A development model for intelligent cities. Paper presented at the 11th International Conference on Computer and Information Technology (CIT-2011) and the 11th IEEE International Conference on Scalable Computing and Communications (ScalCom-2011)

Beijing Social Office. (2013). Beijing guidelines of smart community (trial). Beijing: Beijing Social Office

Deakin, M., \& Al Waer, H. (2011). From intelligent to smart cities. Intelligent Buildings International, 3, 140-152

Hollands, R.G. (2008). Will the real smart city please stand up? City, 12, 303-320

Wright, S., \& Steventon, A. (2004). Intelligent spaces - the vision, the opportunities and the barriers. BT Technology Journal, 22, 15-26

$\mathrm{Wu}$, X., \& Yang, Z. (2010). The concept of the Smart City and future city development. Urban Studies (Edinburgh, Scotland), 11, 56-60

Xu Q., Wu, Z., \& Chen, L. (2012). The vision, architecture and research models of smart city. Journal of Industrial Engineering/ Engineering Management, 26(4): 1-7 